\title{
Efecto analgésico de dos formas farmacéuticas tópicas con extracto de Lactuca sativa
}

\section{Analgesic effect of two topical dosage forms with Lactuca sativa extract}

\author{
Gabriel Sánchez-Cruz ${ }^{1}$ y Héctor Ulises Bernardino-Hernández ${ }^{1}$ \\ Fecha de recepción: 5 de diciembre de 2018 \\ Fecha de aceptación: 5 de marzo de 2019
}

Antony Cabrera-López, ${ }^{1 *}$ Asela Garnica-Sánchez,${ }^{1}$ Leobardo Reyes-Velasco, ${ }^{1}$

\begin{abstract}
Resumen - El dolor es un problema de salud pública. Su tratamiento se realiza mediante fármacos sintéticos convencionales (analgésicos no opioides y opioides), que presentan efectos negativos a nivel hepático, gástrico y renal. Se encuentran en estudio alternativas menos agresivas que consisten en utilizar diversas especies vegetales. Lactuca sativa es una planta empleada como remedio popular para tratar el insomnio, problemas estomacales, dolor e inflamación; sus extractos han sido evaluados farmacológicamente como anticonvulsivantes, sedantes-hipnóticos y antioxidantes, principalmente en modelos animales. Se realizó un estudio para evaluar la propiedad analgésica de dos formas farmacéuticas tópicas elaboradas con extracto de Lactuca sativa, para el tratamiento alternativo en pacientes que presentan dolor muscular agudo. Mediante ensayo clínico controlado y aleatorizado, se evaluó una crema y un gel al $10 \%$ de extracto, se valoró el dolor mediante la Escala de la Expresión Facial de Wong-Baker modificada, así como el grado de alivio y las reacciones adversas. Ambos productos son una alternativa para el tratamiento de dolor agudo frente al uso de fármacos de síntesis convencionales.
\end{abstract}

\section{Palabras clave:}

Lechuga, dolor, extracto, Lactuca sativa.
Abstract - Pain is a public health problem. Its pharmacological treatment is performed through conventional synthetic drugs (non-opioid analgesics and opioids), which have negative effects at the liver, gastric and renal levels. Less aggressive alternatives which use different plant species are under study. Lactuca sativa is a plant used as a popular remedy to treat insomnia, stomach problems, pain and inflammation. Its extracts have been evaluated pharmacologically as anticonvulsants, sedativehypnotics and antioxidants, mainly in animal models. A study was carried out to evaluate the analgesic property of two topical pharmaceutical forms elaborated with Lactuca sativa extract, for the alternative treatment of patients with acute muscular pain. Through a randomized, controlled clinical trial, a cream and gel at 10\% extract were evaluated. Pain was assessed using the modified Wong-Baker Facial Expression Scale at the beginning and end of treatment, as well as the degree of relief and adverse reactions. Both products are an alternative for the treatment of acute pain versus the use of conventional synthetic drugs.

\section{Keywords:}

Lettuce, pain, extract, Lactuca sativa.

$1^{*}$ Facultad de Ciencias Químicas, Universidad Autónoma "Benito Juárez" de Oaxaca. Av. Universidad s/n, col. Cinco Señores, C.P. 68120, Oaxaca de Juárez, Oax., México. *Correo electrónico: elemental_smash@hotmail.com 


\section{Introducción}

E dolor es un síntoma y manifestación clínica subjetiva, multidimensional y compleja que presenta un individuo. Es un fenómeno derivado de la percepción sensorial, emocional y/o cognitiva desagradable de un estímulo eventualmente dañino, asociada a daño tisular real o potencial, incluso sin que haya lesión verificable (Wilson, 2002; Cerveró y Laird, 2002).

Existen diversas formas de clasificar el dolor, las más utilizadas se basan en el mecanismo neurofisiológico (nociceptivo y neuropático, somático y visceral), en el aspecto temporal en función de la duración de los síntomas (agudo y crónico), ${ }^{2}$ en la intensidad del dolor (leve, moderado y severo), en la etiología (por traumas, cáncer, artritis, entre otros) y en la región afectada (cabeza, tórax, espalda, etcétera). En su conjunto tienen una alta prevalencia e importancia en la sociedad actual, por lo que se ha considerado como un problema de salud pública (Jensen et al., 2001; Covarrubias-Gómez, 2008) debido a los impactos negativos que provocan a nivel individual, familiar, laboral, social y económico.

El tratamiento farmacológico del dolor, en cualquiera de sus modalidades, se realiza en tres fases escalonadas de acuerdo con el alivio del paciente: fase 1) analgésicos no opioides con o sin adyuvantes; fase 2) opioides menores con o sin no opioides y adyuvantes; fase 3 ) opioides mayores con o sin no opioides y adyuvantes (Flórez, 2004). Existe una diversidad de fármacos que se utilizan en cualquiera de las tres fases, que han demostrado su eficacia en el tratamiento del dolor; sin embargo, están surgiendo evidencias de sus efectos negativos a nivel hepático, gástrico y renal (García-Andreu, 2017), además de que se encuentran en estudio alternativas menos agresivas.
Al respecto, los usos de diversas plantas medicinales incluidas en la Farmacopea Herbolaria de los Estados Unidos Mexicanos son una opción accesible, en virtud de sus efectos terapéuticos, especialmente en el ámbito de sus propiedades analgésicas. Entre esta diversidad se encuentra la lechuga (Lactuca sativa), que ha sido utilizada en la medicina tradicional de diversas culturas por sus efectos sedantes (Ávila-Uribe, GarcíaZárate, Sepúlveda-Barrera y Godínez-Rodríguez, 2016; Arias, 2009); también se ha reportado como remedio popular para problemas estomacales (estreñimiento, acidez, dispepsia, indigestión y falta de apetito), dolor e inflamación (Araruna y Carlos, 2010).

La lechuga es una herbácea de hoja anual, pertenece a la familia Asteraceae y al género Lactuca. Contiene nutrientes esenciales para el humano, como minerales ( $K$, Na y Ca), vitaminas ( $A, K, E$ y $C$ ) y otros metabolitos importantes (inositol, flavonoides lipofílicos, saponinas, esteroles, polifenoles, ácido rosmarínico y ácido ursólico) (Cho et al., 2009; Nicolle et al., 2004). Además, es rica en quercetina, compuesto con actividad antioxidante (Altunkaya, Becker, Gokmen y Skibsted, 2009), antitrombótica y anticarcinogénica; contiene kaempferol y pequeñas cantidades de miricetina, luteolina, apigenina b-sitosterol, stigmasterol y campesterol, que participan en funciones biológicas como la reducción de los niveles séricos de colesterol y protegen frente a algunos tipos de cáncer (Mahan y Escott, 2009; Nicolle et al., 2004a). Algunos estudios farmacológicos han evaluado las propiedades anticonvulsivantes, sedantes-hipnóticas y antioxidantes de sus extractos crudos (Hefnawy y Ramadan, 2013).

De acuerdo con lo anterior, es necesario profundizar en el análisis de sus propiedades antiinflamatorias y analgésicas, que han sido poco estudiadas, particularmente en la elaboración de

${ }^{2}$ El nociceptivo (normal o sensorial) es el que aparece en todos los individuos normales como consecuencia de la aplicación de estímulos que producen daño o lesión a órganos somáticos o viscerales. El neuropático (anormal o patológico) lo sufren pocos individuos y es resultado de enfermedad o lesión del SNC o periférico. El somático afecta la piel, músculos, articulaciones, ligamentos o huesos; es un dolor localizado, circunscrito a la zona dañada y caracterizado por sensaciones claras y precisas. El visceral afecta órganos internos y aparece regularmente como consecuencia de distintas enfermedades, es un dolor vago, mal localizado y que se extiende más allá de los órganos lesionados. El agudo es consecuencia sensorial inmediata de la activación del sistema nociceptivo, debida generalmente al daño tisular somático o visceral, se desarrolla con un curso temporal que sigue de cerca el proceso de reparación y cicatrización de la lesión causal. El crónico es el que persiste más allá de la lesión que lo originó y que permanece una vez que dicha lesión desaparece (Pedrajas y Molino, 2008). 
formas farmacéuticas apropiadas para su uso en el tratamiento de dolor muscular. El objetivo del presente documento fue evaluar la capacidad analgésica de dos formas farmacéuticas tópicas a partir de extracto de $L$. sativa como medicamento alternativo en pacientes que presentan dolor muscular agudo.

\section{Materiales y métodos}

Se realizó un ensayo clínico controlado y aleatorizado, aprobado por la Comisión de Bioética de la Facultad de Ciencias Químicas de la Universidad Autónoma "Benito Juárez" de Oaxaca (UABJO). Todos los pacientes firmaron el consentimiento informado para llevar a cabo el estudio, que duró cuatro meses, de enero a abril de 2018.

Se evaluaron dos tratamientos tópicos aplicados sobre la piel en pacientes que presentaban dolor agudo: gel al $10 \%$ de extracto de L. sativa, crema al $10 \%$ del mismo extracto y como control se utilizó diclofenaco dietilamonio en gel al $1.16 \%$. El gel y la crema se prepararon previamente a partir del extracto concentrado acuoso grado cosmético de $L$. sativa, adquirida a la empresa Bioextracto S.A. de C.V. La crema se elaboró a base de ácido esteárico, esperma de ballena, cera de abeja, aceite de ajonjolí, agua de rosas y extracto. El gel se preparó con agua destilada, carbopol, benzoato de sodio, etanol y extracto. El diclofenaco se adquirió en una farmacia comercial y fue de marca genérica. No se utilizaron controles con grupos placebo, debido a que se contó con un tratamiento adecuado a partir de diclofenaco. Los excipientes empleados no poseen propiedades analgésicas, por lo que no influyeron en el efecto investigado.

Se consideraron como criterios para participar en este estudio contar con la mayoría de edad y presentar al momento del inicio dolor muscular de tipo agudo derivado de algún trauma o ejercicio prolongado. Se excluyó a los individuos con hipersensibilidad a la lechuga, con dolor crónico de cualquier tipo, con heridas en la región con dolor, con patologías reumáticas, diabetes, insuficiencias orgánicas, obesidad mórbida y que estuvieran bajo tratamiento de tipo tópico.

Participaron en el estudio 39 personas (28 hombres y 11 mujeres), entre 18 a 62 años (media de 26.4 años), todas previamente informadas y con consentimiento firmado. Cada individuo fue aleatorizado para recibir uno de los tres tratamientos; a todos se les capacitó para utilizar el tratamiento correspondiente. La dosis fue de una porción del tamaño de la yema de su dedo medio, aplicada mediante frotación en el área con dolor hasta su absorción total, cada 8 horas mientras persistiera el dolor o por un máximo de siete días de aplicación. La valoración del dolor se realizó mediante la Escala de la Expresión Facial (EEF de Wong-Baker), modificada para seleccionar valores entre 0 a $5(0=$ ausencia de dolor, $1=$ dolor leve, 2 y $3=$ dolor moderado, $4=$ dolor intenso y $5=$ dolor insoportable 0 máximo dolor imaginable) ${ }^{3}$ (Montero y Manzanares, 2005). La medición se efectuó al inicio y al final del tratamiento. Se consideró como grado de alivio la diferencia entre el valor inicial y el valor final, además de contabilizar el total de días en que se logró la desaparición del dolor y se complementó con las manifestaciones de reacciones adversas percibidas por los usuarios con el uso de cualquiera de los tratamientos. Para identificar las diferencias entre éstos se realizó un análisis de varianza y prueba post hoc de Scheffe y Games Howeell para varianzas homogéneas y heterogéneas, respectivamente. El análisis se hizo con el software SPSS v15.0 para Windows.

\section{Resultados}

La crema exhibió una consistencia homogénea, con textura cremosa, coloración blanca, olor suave a rosas y sabor amargo. Por su parte, el gel presentó una consistencia suave y homogénea, incolora, olor fuerte

${ }^{3}$ La Escala de la Expresión Facial (Faces Pain Scale) es usada en niños y está compuesta por rostros con diferentes expresiones que representan a una persona que está feliz porque no siente dolor o que está triste porque siente diferentes niveles de dolor. $0=$ muy contento, sin dolor; $2=$ siente sólo un poquito de dolor; $4=$ siente un poco más de dolor; $6=$ siente aún más dolor; $8=$ siente mucho dolor y $10=$ el dolor es el peor que puede imaginarse (no necesariamente tiene que haber llanto por sentir este dolor tan fuerte). 
a etanol y sabor agrio. Las dos formas farmacéuticas propuestas son estables a un tiempo aproximado de tres meses.

Los participantes en el estudio sufrían principalmente dolor en extremidades inferiores $(36.0 \%$ en el muslo, $17.9 \%$ en alguna rodilla y $12.8 \%$ en un tobillo), seguido de la espalda (15.4\%) y extremidades superiores $(5.1 \%$ brazo y codo, respectivamente, $5.1 \%$ mano y $2.6 \%$ en algún hombro).

No se registraron diferencias significativas en la presencia de dolor entre los grupos antes de iniciar los tratamientos. El promedio de dolor inicial fue de $3.4 \pm 0.7 ; 64.1 \%$ manifestó dolor moderado, $23.1 \%$ dolor intenso, $10.3 \%$ dolor insoportable y solamente una persona manifestó dolor un poco más que leve (2.6\%). Después de la aplicación de los tres tratamientos no se observaron diferencias en la percepción de dolor. Los tres tratamientos lo eliminaron totalmente en una proporción similar de individuos (aproximadamente $50 \%$ ). Con respecto al grado de alivio, no se detectaron diferencias entre los tres tratamientos, disminuyendo de manera semejante las puntuaciones que van de dolor intenso-moderado a dolor leve y alivio total (cuadro 1). Los pacientes reportaron una mejoría alrededor de tres días posteriores al inicio del tratamiento con cualquiera de los tres evaluados.

El gel y la crema a base de extracto de Lactuca sativa tuvieron el mismo efecto analgésico y antiinflamatorio que el diclofenaco. Ninguno de los individuos tratados manifestó haber experimentado alguna reacción adversa (prurito, eritema, pérdida de sensibilidad, entre otros) por la aplicación de los productos a base del extracto vegetal.

\section{Discusión}

La crema y el gel al $10 \%$ de extracto de Lactuca sativa mostraron resultados promisorios como analgésicos y antiinflamatorios tópicos que pudieran constituir una estrategia alternativa para el tratamiento del dolor agudo, frente a fármacos sintéticos convencionales. La aplicación tópica de analgésicos ejerce sus efectos y posibilita elevadas concentraciones en los sitios efectores periféricos, en contraposición con sus bajos niveles séricos (McCleane, 2007). De esta manera, representan una opción terapéutica asociada a menor incidencia de efectos colaterales no deseados, en comparación con la administración vía sistémica de los mismos productos (Pereira, Carvalho y Dos Santos, 2012).

La evidencia científica disponible sobre la eficacia clínica de extractos vegetales en formas farmacéuticas de tipo tópico en humanos, particularmente de la lechuga, es escasa; la mayoría de los estudios que han evaluado sus propiedades analgésicas y antiinflamatorias se han realizado en modelos animales, específicamente en ratones. Al respecto, Sayyah, Hadidi y Kamalinejad (2004) demostraron los resultados antinociceptivos y antiinflamatorios del extracto crudo en ratas, sin mostrar comportamientos anormales o letalidad. El análisis fitoquímico preliminar reveló la presencia de triterpenoides, saponinas y fenoles simples en el extracto. Por su parte, Ismail y Mirza (2015) observaron la reducción de dolor e inflamación en ratas cuando se les administró la mezcla de los extractos de semilla y hoja de lechuga, además de inducir un efecto anticoagulante, sin que presentaran ningún comportamiento anormal o letalidad en algún animal probado.

Aunque no se identificaron los metabolitos relacionados con los efectos analgésicos en el presente estudio, diversas investigaciones han reportado distintos activos asociados a dichas propiedades. García y GómezReino (2000) hallaron que las antocianinas [cianidin3-0- (6 "-malonil- $\beta$-glucopiranósido) y cianidin-3-0- (6" -malonil- $\beta$-glucopiranósido éster metílico)], así como el ácido chicórico, quercetina glucósido, ácido ferúlico y cafeico del extracto de Lactuca sativa roja, inhibieron la peroxidación lipídica (LPO), también las enzimas COX1 y 2 (Mulabagal et al., 2010), que intervienen en el proceso de dolor e inflamación. Wesołowska, Nikiforuk, Michalska, Kisiel y Chojnacka-Wójcik (2006) estudiaron a la lechuga silvestre (Lactuca virosa) y hallaron que contiene lactonas sesquiterpénicas (lactucina y sus derivados, lactucopicrina y 11-beta, 13-dihidrolactucina), responsables de los efectos analgésicos y sedantes. Por 
pertenecer al mismo género de la Lactuca sativa, se sospecha que contienen los mismos activos.

Los pacientes que recibieron los tratamientos con el extracto vegetal no manifestaron ninguna reacción adversa. En todos los estudios mencionados anteriormente, que utilizaron animales de experimentación, éstos no presentaron comportamientos anormales o letalidad. Aunque no es comparable con pacientes humanos, insinúa la posibilidad de riesgos mínimos al utilizarse como tratamientos tópicos; sin embargo, no debe descartarse el riesgo de alergias, que aunque son aisladas, pudieran sobrevenir. Al respecto, Muñoz-García et al. (2013) encontraron que la traumatina y la aspartil proteasa son alérgenos de la lechuga y las reacciones alérgicas ocurren en el contexto del síndrome de sensibilización a una proteína de transferencia de lípidos (LTP), siendo el melocotón el sensibilizador primario y los pólenes en la mayoría de los casos (MuñozGarcía et al., 2017). En pruebas posteriores, un criterio para excluir a los individuos podría ser la alergia a dichos sensibilizadores.

Una limitante del estudio fue el reducido tamaño de la muestra. Se sugiere que a futuro se incremente el número de individuos y se contemple la influencia de las variables edad, sexo, tipo de dolor (agudo y crónico), entre otros, en la respuesta al tratamiento con el extracto. Finalmente, hay mucho por investigar en relación con las propiedades analgésicas de la lechuga, tales como su toxicidad y letalidad en modelos in vitro e in vivo, los activos asociados a la actividad analgésicaantiinflamatoria y su mecanismo de acción, la evaluación de otras concentraciones de la misma u otras formas farmacéuticas, la adhesión al tratamiento, la duración de la analgesia, la tolerancia a largo plazo, la satisfacción del paciente, entre otros.

\section{Conclusiones}

La crema y gel al $10 \%$ de extracto de L. sativa son una alternativa para tratar el dolor agudo frente al uso de fármacos de síntesis convencionales. Es importante continuar con los estudios científicos de diversas especies vegetales, con la finalidad de acumular evidencias que garanticen la seguridad de su uso en la medicina tradicional, así como en la elaboración de formas farmacéuticas que contribuyan en tratamientos alternativos en beneficio de la salud de los pacientes.

\section{Referencias}

Altunkaya, A., Becker, E. M., Gokmen, V. y Skibsted, L. H. (2009). Antioxidant activity of lettuce extract (Lactuca sativa) and synergism with added phenolic antioxidants. Food Chemistry, 115(1), 163-168. Disponible en https://www.researchgate.net/ publication/221962407_Antioxidant_activity_of_ lettuce_extract_Lactuca_sativa_and_synergism_ with_added_phenolic_antioxidants

Araruna, K. y Carlos, B. (2010). Anti-inflammatory activities of triterpene lactones from Lactuca sativa. Phytopharmacology, 1(1), 1-6. Disponible en http://citeseerx.ist.psu.edu/viewdoc/ download?doi=101.1.207.9101\&rep=rep1\&type=pdf

Arias T., B. (2009). Diversidad de usos, prácticas de recolección y diferencias según género y edad en el uso de plantas medicinales en Córdoba, Argentina. Boletín Latinoamericano y del Caribe de Plantas Medicinales y Aromáticas, 8(5), 389401. Disponible en https://www.redalyc.org/ pdf/856/85611977005.pdf

Ávila-Uribe, M. M., García-Zárate, S. N., SepúlvedaBarrera, A. S. y Godínez-Rodríguez, M. A. (2016). Plantas medicinales en dos poblados del municipio de San Martín de las Pirámides, Estado de México. Polibotánica, 42, 215-245. Recuperado de http://www.scielo. org.mx/scielo.php?script=sci_abstract\&pid=\$140527682016000200215\&lng=es\&nrm=iso

Cerveró, F. y Laird J., M. A. (2002). Fisiología del dolor. En L. Aliaga L., J. E. Baños, C. de Barutell, J. Molet y D. A. Rodríguez (eds.), Tratamiento del dolor. Teoría y práctica (pp. 9-25). Barcelona: P. Permanyer, S.L. $2^{a}$ ed.

Cho, W. K., Chen, X. Y., Uddin, N. M., Rim, Y., Moon, J., Jung, J. H., Shi, C., Chu, H., Kim, S., Kim, S. W., Park, Z. Y. y Kim, J. Y. (2009). Comprehensive proteome analysis of lettuce latex using multidimensional protein-identification 
technology. Phytochemistry, 70(5), 570-578. Disponible en https://www.ncbi.nlm.nih.gov/pubmed/19356777

Covarrubias-Gómez, A. (2008). Papel del anestesiólogo en el manejo del dolor crónico. Rev Mex Anest., 31(Suppl. 1), S235-S237. Disponible en http://www.medigraphic. com/pdfs/rma/cma-2008/cmas081ba.pdf

Flórez, J. (2004). Fármacos y dolor. Madrid: Ed. Ergon.

GarcíaM.J.J.A.y Gómez-ReinoC.,J.J.(2000).Fisiopatología de la ciclooxigensasa-1 y ciclooxigenasa-2. Revista Española de Reumatología, 27(1), 33-35. Disponible en http://www.elsevier.es/es-revista-revistaespanola-reumatologia-29-articulo-fisiopatologiaciclooxigenasa-1-ciclooxigenasa-2-8546

García-Andreu, J. (2017). Manejo básico del dolor agudo y crónico. Anestesia en México, 29(Supl.1), 7785. Disponible en de http://www.scielo.org.mx/pdf/ am/v29s1/2448-8771-am-29-00077.pdf

Hefnawy, T. M. y Ramadan, M. F. (2013). Protective effects of Lactuca sativa ethanolic extract on carbon tetrachloride induced oxidative damage in rats. Asian Pacific J Trop Dis., 3, 277-285. Disponible en https:// www.ncbi.nlm.nih.gov/pmc/articles/PMC4027310/

Ismail, H. y Mirza, B. (2015). Evaluation of analgesic, anti-inflammatory, anti-depressant and anti-coagulant properties of Lactuca sativa (CV. Grand Rapids) plant tissues and cell suspension in rats. Complementary and Alternative Medicine, 15(199). Disponible en https:// www.ncbi.nlm.nih.gov/pmc/articles/PMC4482268/

Jensen, T. S., Gottrup, $H_{\text {., }}$ Kasch, $H_{\text {., Nikolajsen, }}$ L., Terkelsen, A. J. y Witting, N. (2001). Has basic research contributed to chronic pain treatment? Acta Anaesthesiol Scand, 45(9), 1128-1135. Disponible en https://onlinelibrary.wiley.com/doi/epdf/10.1034/ j.1399-6576.2001.450913.x

Mahan, K. y Escott, S. (2009). Krause Dietoterapia. España: Elservier Masson. Disponible en http://www. sennutricion.org/es/2009/02/25/krause-dietoterapiaincluye-evolve-12-ed

McCleane, G. (2007). Topical analgesics. Anesthesiol Clin., 25, 825-839. Disponible en http://www.scielo.br/scielo.php?script $=s c i$ n links\&ref = $000075 \& p i d=S 0034$ $7094201200020001000001 \&$ lng=en
Montero I., R. y Manzanares, B. A. (2005). Escalas de valoración del dolor. Diagnóstico, LXVIII(1.553), 529-530. Disponible en https://www.academia. edu/28244472/Escalas_de_valoraci\%C3\%B3n_ del_dolor

Mulabagal, V., Ngouajio, M., Nair, A., Zhang, Y., Gottumukkala, A. L. y Nair, M. G. (2010). In vitro evaluation of red and green lettuce (Lactuca sativa) for functional food properties. Food Chemistry, 118(2), 300-306. Disponible en https:// www.extension.iastate.edu/vegetablelab/files/ research/files/In\%20vitro\%20evaluation $\% 20$ of $\% 20$ red $\% 20$ and $\% 20$ green $\% 2$ Olettuce $\% 20$ (Lactuca\%20sativa)\%20for\%20functional\%20 food\%20properties.pdf

Muñoz-García, E., Luengo-Sánchez, O., Haroun-Díaz, E., Sanz, M. A., Palacin, A., Díaz-Perales, A., Heras, G. M., Labrador-Horrillo, M., Vivanco, F., CuestaHerranz, J. y Pastor-Vargas, C. (2013). Identification of thaumatin-like protein and aspartyl protease as new major allergens in lettuce (Lactuca sativa). Mol. Nutr. Food Res., 57, 2245-2252. Disponible en https://www.ncbi.nlm.nih.gov/pubmed/27609533

Muñoz-García, E., Luengo-Sánchez, O., MorenoPérez, N., Cuesta-Herranz, J., Pastor-Vargas, C. y Cardona, V. (2017). Lettuce allergy is a lipid transfer syndrome-related food allergy with a high risk of severe reactions. I Investig Allergol Clin Immunol, 27(2), 98-103. Disponible en http://www.jiaci.org/ revistas/vol27issue2_3.pdf

Nicolle, C., Carnat, A., Fraisse, D., Lamison, J. L., Rock, E., Michel, H., Amouroux, P. y Remesy, C. (2004). Characterization and variation of antioxidant micronutrients in lettuce (Lactuca sativa folium). J. Sci. Food Agric., 84(15), 2061-2069. Disponible en https://onlinelibrary.wiley.com/doi/abs/10.1002/ jsfa.1916

Nicolle, C., Cardinault, N., Gueux, E., Jaffrelo, L., Rock, E., Mazur, A., Amouroux, P. y Rémesy, C. (2004a). Health effect of vegetable-based diet: lettuce consumption improves cholesterol metabolism and antioxidant status in the rat. Clin Nutr., 23, 605614. Disponible en https://www.ncbi.nIm.nih.gov/ pubmed/15297097

Pedrajas N., J. M. y Molino G., Á. M. (2008). Bases neuromédicas del dolor. Clínica y Salud, 19(3), 277- 
293. Disponible en http://scielo.isciii.es/pdf/clinsa/ v19n3/v19n3a02.pdf

Pereira F., M.; Carvalho R., A. P. y Dos Santos N., J. (2012). Analgésicos Tópicos. Rev Bras Anestesiol., 62(2), 244-252. Disponible en http://www.scielo.br/pdf/rba/ v62n2/es_v62n2a10.pdf

Sayyah, M., Hadidi, N. y Kamalinejad, M. (2004). Analgesic and anti-inflammatory activity of Lactuca sativa seed extract in rats. J Ethnopharmacol, 92(2-3), 325-329. Disponible en https://www.ncbi.nlm.nih.gov/ pubmed/15138019
Wesołowska, A., Nikiforuk, A., Michalska, K., Kisiel, W. y Chojnacka-Wójcik, E. (2006). Analgesic and sedative activities of lactucin and some lactucin-like guaianolides in mice. J Ethnopharmacol, 107(2), 254-258. Disponible en https://www.ncbi.nlm.nih.gov/ pubmed/16621374

Wilson, K. E. (2002). Implicaciones psicológicas del dolor crónico. En P. Prithvi Raj (coord.), Tratamiento práctico del dolor (pp. 332-346). Madrid: Edide S.L., $3^{\text {a ed. }}$

\section{Cuadro 1.}

Presencia de dolor antes y después de la aplicación de los tratamientos

\begin{tabular}{|c|c|c|c|}
\hline & Diclofenaco & Gel $10 \%$ & Crema 10\% \\
\hline \multicolumn{4}{|l|}{ Nivel del dolor antes del tratamiento } \\
\hline Promedio $\pm \mathrm{DE}$ & $3.3 \pm 0.5 a$ & $3.1 \pm 0.7 a$ & $3.8 \pm 0.8 a$ \\
\hline Amplitud & $3-4$ & $2-5$ & $3-5$ \\
\hline IC para la media al 95\% & $3.0-3.6$ & $2.7-3.6$ & $3.3-4.3$ \\
\hline \multicolumn{4}{|l|}{ Frecuencia (No. de individuos) } \\
\hline 2 (dolor un poco más que leve) & & 1 & \\
\hline 3 (dolor moderado) & 9 & 10 & 6 \\
\hline 4 (dolor intenso) & 4 & 1 & 4 \\
\hline 5 (dolor insoportable) & & 1 & 3 \\
\hline \multicolumn{4}{|l|}{ Nivel de dolor después del tratamiento } \\
\hline Promedio $\pm \mathrm{DE}$ & $0.8 \pm 0.9 a$ & $0.5 \pm 0.7 a$ & $0.5 \pm 0.5 a$ \\
\hline Amplitud & $0-2$ & $0-2$ & $0-1$ \\
\hline IC para la media al 95\% & $0.3-1.4$ & $0.1-0.9$ & $0.1-0.8$ \\
\hline \multicolumn{4}{|l|}{ Frecuencia (No. de individuos) } \\
\hline 0 (sin dolor) & 6 & 7 & 7 \\
\hline 1 (dolor leve) & 3 & 5 & 6 \\
\hline 2 (dolor moderado) & 4 & 1 & -- \\
\hline \multicolumn{4}{|c|}{ Grado de alivio (dolor inicial - dolor final) } \\
\hline Promedio $\pm \mathrm{DE}$ & $2.5 \pm 1.1 \mathrm{a}$ & $2.6 \pm 0.8 a$ & $3.2 \pm 1.0 a$ \\
\hline Amplitud & $1-4$ & $1-4$ & $2-5$ \\
\hline IC para la media al 95\% & $1.8-3.1$ & $2.2-3.1$ & $2.6-3.8$ \\
\hline \multicolumn{4}{|l|}{ Frecuencia (No. de individuos) } \\
\hline 0 (no disminuyo el dolor) & -- & -- & -- \\
\hline 1 (disminuyó un punto el dolor) & 3 & 1 & -- \\
\hline 2 (disminuyó dos puntos el dolor) & 3 & 4 & 3 \\
\hline 3 (disminuyo tres puntos el dolor) & 5 & 7 & 6 \\
\hline 4 (disminuyó cuatro puntos el dolor) & 2 & 1 & 2 \\
\hline 5 (disminuyó cinco puntos el dolor) & -- & -- & 2 \\
\hline
\end{tabular}




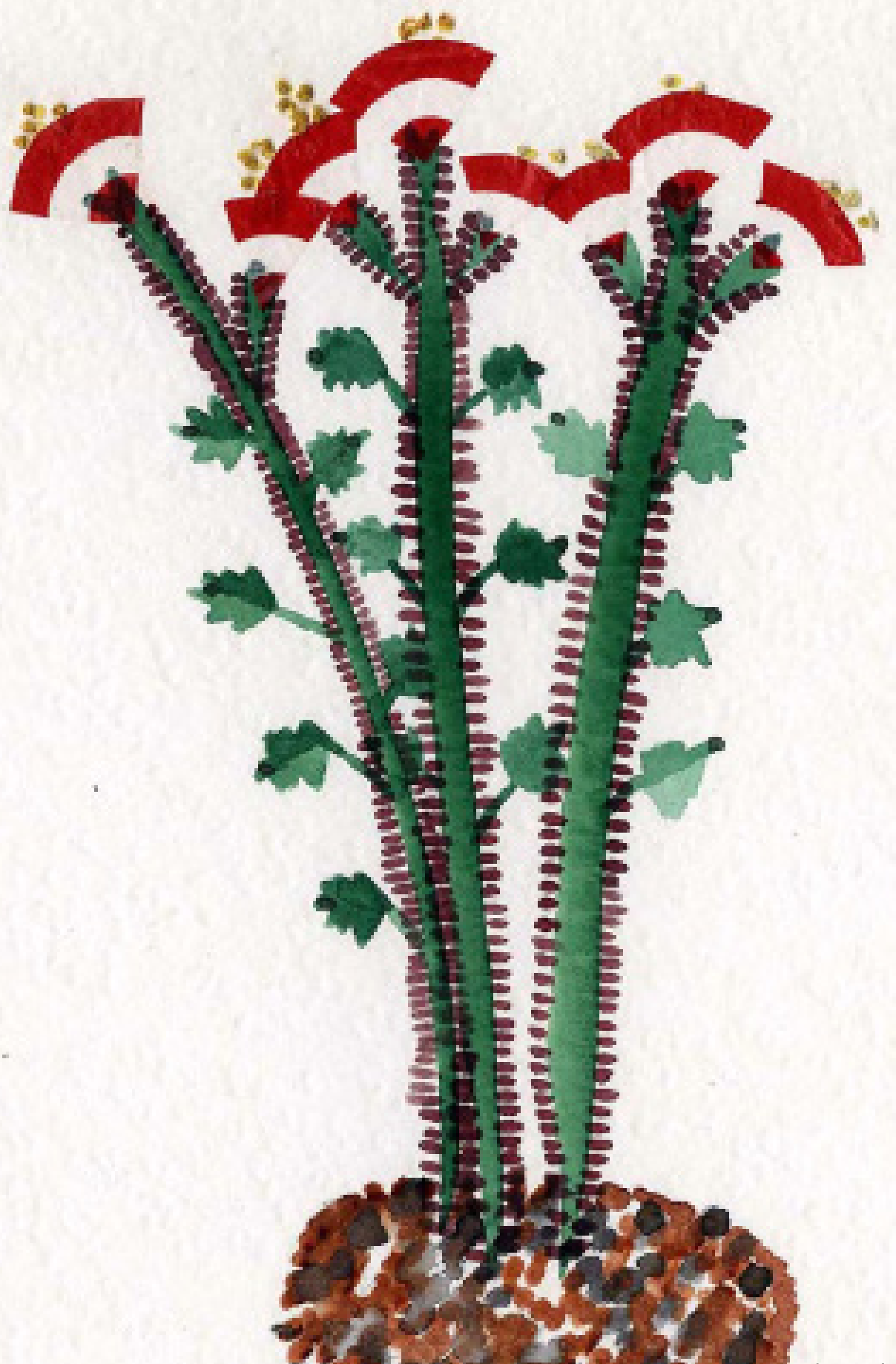

"Quauhalahuac target" Acuarela, fragmentos de bolsas de papel de productos de consumo y bordado sobre papel de algodón. $26 \times 18 \mathrm{~cm}$ 


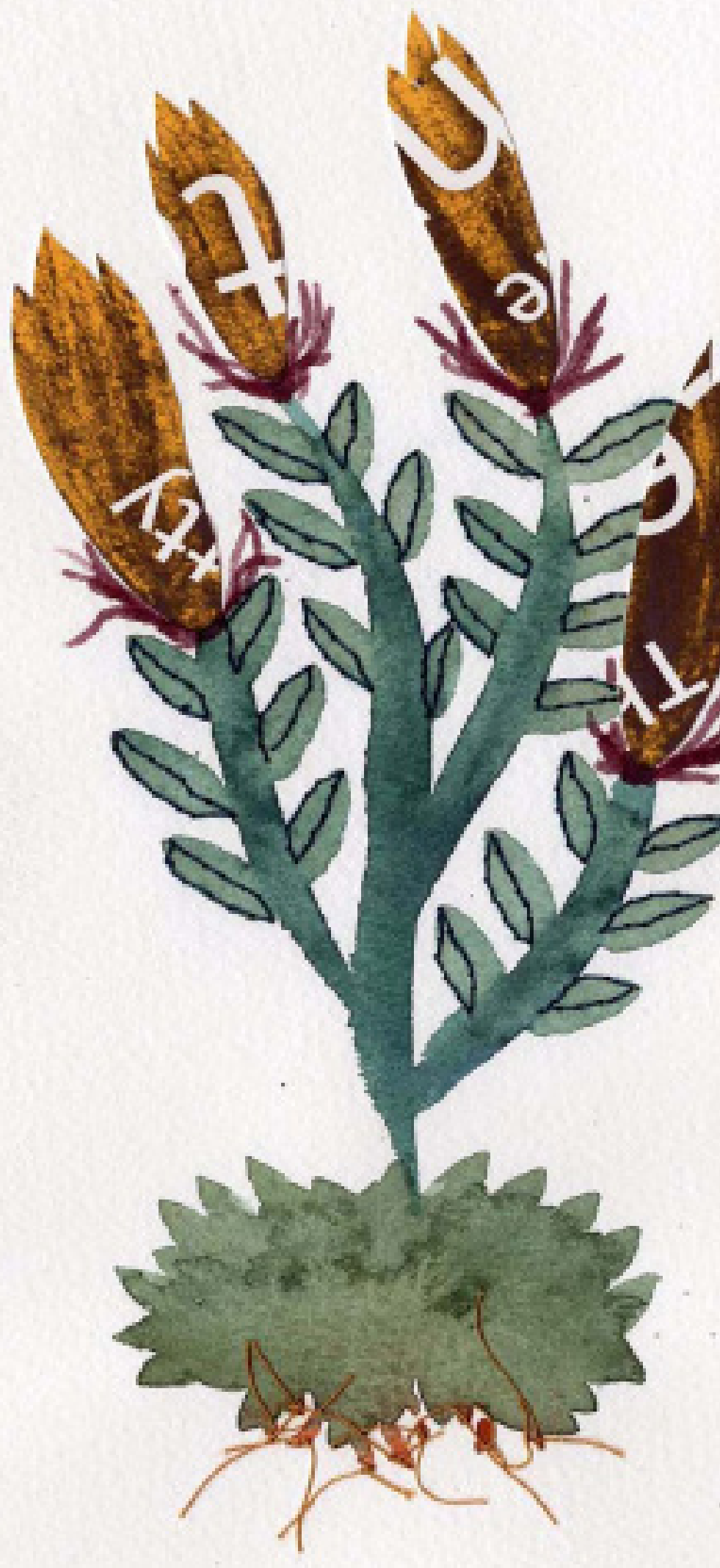

"Cacaloxochitl getty" Acuarela, fragmentos de bolsas de papel de productos de consumo y bordado sobre papel de algodon. $26 \times 18 \mathrm{~cm}$ 